\title{
Can we perform adjuvant chemotherapy alone in stage IB/IIA cervical cancer meeting Sedlis criteria?
}

\section{Meng Li}

Shandong University Qilu Hospital

\section{Ying Gu}

Shandong University Qilu Hospital

Yawen Zheng

Shandong University Qilu Hospital

Xiaohang Yang

Shandong University Qilu Hospital

\section{Baoxia Cui}

Shandong University Qilu Hospital

\section{Bin Wu}

Taian City Central Hospital

Xingsheng Yang ( $\nabla$ xingshengyang@sdu.edu.cn )

Department of obstetric and gynecology『Qilu hospital『Shandong University https://orcid.org/00000002-4946-2033

\section{Research article}

Keywords: Cervical cancer; Chemotherapy; Radiotherapy; Sedlis criteria

Posted Date: August 20th, 2019

DOI: https://doi.org/10.21203/rs.2.13188/v1

License: (c) (1) This work is licensed under a Creative Commons Attribution 4.0 International License.

Read Full License 


\section{Abstract}

Background: The aim of this study was to evaluate the effectiveness of chemotherapy alone as postsurgical adjuvant therapy on survival of patients in stage IB/IIA cervical squamous cell carcinoma meeting Sedlis criteria. Methods: We retrospectively reviewed the records of 127 patients satisfying Sedlis criteria after radical surgery for stage IB/IIA cervical cancer from January 2009 to December 2013. Of these, 65 patients received radiotherapy (RT)/chemoradiotherapy (CRT), 62 patients received chemotherapy (CT) alone. Both the treatment modalities were compared on 5-year overall survival (OS), disease-specific survival (DSS), recurrence rates and side effects. Results: Median follow-up time for surviving patients was 75 months. The 5-year OS was similar in both the groups with $85.5 \%$ in the CT and $87.7 \%$ in the RT/CRT group, respectively, $(\mathrm{p}=0.727)$. Likewise, there was no significant difference in 5-year DSS between the two arms (CT: $87.1 \%$ vs. RT/CRT: $90.8 \% ; \mathrm{P}=0.548$ ). Rate and the pattern of recurrence were not different between the groups. Acute toxicity was more frequent in the CT group, but was statistically insignificant $(P>0.05)$. The incidences of urinary disturbance $(P=0.003)$ and lymphedema $(P=0.041)$ were fewer in the CT group. Conclusions: Adjuvant CT alone for early-stage cervical cancer patients who meet the Sedlis criteria might be a good alternative to RT/CRT.

\section{Background}

Globally, cervical cancer is the fourth most frequent cancer among women, with an estimated 570000 new cases and 311,000 deaths in 2018. Underdeveloped countries carry a significant proportion (more than $85 \%$ ) of the burden of deaths due to cervical cancer [1]. Radical hysterectomy (RH) with pelvic lymphadenectomy is considered the optimal treatment paradigm for early-stage cervical cancer. Selection of postoperative adjuvant therapy depends on the presence of risk factors associated with recurrence which are affirmed by pathological criteria. Adjuvant radiotherapy (RT) or chemoradiotherapy (CRT) is considered appropriate strategy for preventing recurrence of early-stage cervical cancer post operation [2]. For high-risk postsurgical patients, adjuvant concurrent chemoradiotherapy (CCRT) can improve overall survival rate (OS) and relapse-free survival rate (RFS) [3]. With respect to intermediate-risk patients, previous Gynecologic Oncology Group prospective trial (GOG 92) reported that adjuvant pelvic radiotherapy following radical surgery (RS) dramatically reduced the number of recurrences and prolonged progression-free survival (PFS) in Stage IB cervical cancer patients [4,5]. Although a few studies have reported RT given as an adjuvant therapy decreased pelvic recurrences [6,7], the benefit on overall survival is still debatable $[8,9,10,11]$. On the other hand, radical surgery followed by RT was severely toxic and included adverse events such as bowel obstruction, lymphedema and urinary disturbance $[12,13]$, which distinctly reduced the quality of life $(\mathrm{QOL})$ in early-stage cervical cancer patients $[14,15]$.

Several reports suggested that adjuvant chemotherapy (CT) alone after radical surgery had similar therapeutic effects as RT [16]. No difference in disease-free survival (DFS) or OS has emerged between the two treatment groups. Paclitaxel/cisplatin (TP) combination is regarded as a main chemotherapy regimen by GOG for women in locally advanced or persistent cervical cancer [17]. Nevertheless, CT alone 
has not been widely used as postoperative adjuvant therapy in early-stage cervical cancer. Moreover, there is no clear consensus on specific chemotherapeutic drug and dosage in early-stage cervical cancer. To address these issues, we retrospectively investigated oncologic results in 127 patients meeting the Sedlis criteria to evaluate the impact of CT and ascertain the group that might benefit from this treatment after RS for stage IB/IIA cervical cancer.

\section{Methods}

We retrospectively reviewed the clinical data from 127 patients undergoing treatment from January 2009 to December 2013 in Qilu hospital, Shandong University. This study protocol was approved by the Ethics Committee of Shandong University(KYLL-2018-174). The Ethics Committee of Shandong University approved the Waiver of Consent Requirements for this research. Characteristics of the patients, treatment regime, therapeutic reaction, follow-up on the development of disease and date of death were extracted from electronic medical records. Inclusion criteria were: patients with FIGO (International Federation of Gynecology and Obstetrics) stage IB/IIA cervical cancer; patients with radical hysterectomy and pelvic lymphadenectomy (RH-PLND) before adjuvant therapy; pathology certified as squamous cell carcinoma (SCC); patients satisfying at least one of the Sedlis criteria.: 1) positive lymphovascular space invasion (LVSI), deep one-third stromal invasion plus any tumor size; 2) positive LVSI, middle one-third stromal invasion plus tumor size $\geq 2 \mathrm{~cm}$; 3 ) positive LVSI, superficial one-third stromal invasion plus tumor size $\geq 5 \mathrm{~cm}$; and 4) negative LVSI, deep or middle one-third stromal invasion plus tumor size $\geq 4 \mathrm{~cm}$. Exclusion criteria were as follows: patients with high-risk for recurrence (parametrial invasion, pelvic lymph node metastasis and positive resection margin); patients who accepted neoadjuvant chemotherapy; patients with inconsistent follow-up data or lost follow-up.

Adjuvant CT was administered within 4 weeks after radical surgery. The treatment regimen was paclitaxel combined with cisplatin or carboplatin administered every three weeks. Paclitaxel/cisplatin consists of paclitaxel $\left(175 \mathrm{mg} / \mathrm{m}^{2}\right)$ plus cisplatin $\left(75 \mathrm{mg} / \mathrm{m}^{2}\right)$ triweekly, and paclitaxel/carboplatin (TC) composes of paclitaxel $\left(175 \mathrm{mg} / \mathrm{m}^{2}\right)$ plus carboplatin with the dose of AUC (area under the curve) 5 every 3 weeks. Ninety-five patients (33 patients in the RT/CRT group; 62 patients in the CT group) received adjuvant CT for 2 to 6 cycles (median: 4 cycles) according to the gynecologist's preference, patients' tolerance and response. Detailed CT schedule is shown in Figure 1. Adjuvant RT for the entire pelvic was external irradiation beginning within the 4-6 weeks interval after radical surgery. Each patient received daily fractions of $1.80-2.00 \mathrm{~Gy}$ for a total of 25 fractions ( $50 \mathrm{~Gy}$ ) or a total of 28 fractions ( $50.4 \mathrm{~Gy}$ ).

Endpoints included assessment of 5-year OS and disease-specific survival (DSS). Follow up was every 3 months in the first year, every 6 months in the first five years and annually thereafter. Patients were regularly examined with abdominal or vaginal ultrasounds, pelvic CT and a Papanicolaou smear from vaginal vault. The results of these clinical surveillance, treatment-related toxicity and treatment failure was recorded at the time of each judgement. Assessment of chemotherapy-induced toxicity was done according to the World Health Organization (WHO) guidelines. Late toxicities were evaluated by the RTOG (Radiation Therapy Oncology Group) and EORTC (European Organization for Research and Treatment of 
Cancer) radiation criteria [18]. OS was defined as the interval from the date of primary surgery to the date of death. DSS was determined as the interval from the date of operation to cervical cancer-related death. Estimated OS and DSS were calculated by the Kaplan-Meier survival curves and compared using the log rank test. Pearson's chi-square test was employed to assess the distribution for recurrences and adverse effects in the two arms. Comparison of categorical variables such as clinical stage, Sedlis criteria, and grade were performed by Fisher exact test. $P<0.05$ was deemed as significant. All end points were last updated in December 2018 and statistically analyzed using SPSS 20.0 (SPSS Inc., Chicago, IL, USA).

\section{Results}

At the time of this report, the median age of patients at diagnosis was 47 years (range 24 to 73 years). Patient and tumor characteristics are shown in Table 1. Among the 127 patients, 65 patients received RT/CRT, 62 patients underwent only CT. There were no obvious differences between the arms in several clinical and pathologic variables including: age $(P=0.618)$, clinical stage $(P=0.382)$, Sedlis criteria $(P=0.811)$, and grade $(P=0.792)$. Twenty-six patients $(20.5 \%)$ were at stage IB1, 64 patients $(50.4 \%)$ at IB2, and 6 patients $(4.7 \%)$ at IIA1, 31 patients $(24.4 \%)$ as IIA2. No patient met the third category in Sedlis criteria (positive lymphovascular space invasion, superficial one-third stromal invasion plus tumor size $\geq 5 \mathrm{~cm}$ ). Based on modified Broders' classification, more than half of the patients $(64.6 \%)$ presented with poorly differentiated pathology throughout the study.

Median follow-up time for surviving patients was 75 (range, 60-119) months. At the final follow-up date, a total of 19 patients had died,10 in the CT-only group and 9 in the RT/CRT group. Of these deceased patients, two patients survived for more than five years (one in each group). The estimated 5-year OS in all the patients was $86.6 \%(110 / 127)$; was $85.5 \%(53 / 62)$ in the CT only group and was $87.7 \%(57 / 65)$ in the RT/CRT group, respectively $(\mathrm{P}=0.727)$ (Figure 2). Two patients in the CT group died of cardiovascular disease (one died within five years and the other died more than five years after surgery) and one died of lung carcinoma. In the RT/CRT group, one patient committed suicide and the other died of complication of asthma. The 5-year DSS of the entire patient cohort was estimated to be $89.8 \% ; 88.7 \%$ in the CT arm and $90.8 \%$ in the RT/CRT arm, respectively. Figure 3 shows the DSS survival curves in the CT and RT/CRT groups with no appreciable difference $(\mathrm{P}=0.883)$.

We observed treatment failure in 8 patients $(12.9 \%)$ receiving only CT and $7(10.8 \%)$ patients receiving RT/CRT ( $\mathrm{P}=0.787$ ). In the CT group, loco-regional recurrences were observed in 5 patients (two stumps, three pelvis) and distant metastases in 3 (one lung, one bone, one liver) out of the 8 patients after treatment failure. Loco-regional metastasis occurred in 3 patients (one stump, two pelvis) and distant metastasis occurred in 4 patients (one lung, two bone, one paraaortic lymph node) in the RT/CRT group (Table 2). Rate and pattern of recurrence were similar in both the groups. One patient in the CT group with local recurrence survived after radiation treatment. Despite aggressive treatment, none of the relapsed patients survived in the RT/CRT group. 
No treatment-related deaths occurred during the follow-up. Most of the adjuvant CT related toxicities were transient, tolerable and recoverable by conservative treatment. Acute toxicities included acute grade III to IV hematological toxicity (neutropenia, thrombocytopenia, and anemia), grade III to IV hepatic toxicity and gastrointestinal toxicity (nausea, vomiting, diarrhea), which were non-significantly more frequent in the CT group $(P>0.05)$. Late toxicities were urinary disturbance, lower-limb lymphedema and bowel obstruction. As shown in Table 3, there were fewer occurrences of urinary disturbance $(P=0.003)$ and lower-limb lymphedema $(P=0.041)$ in the CT group as compared to the RT/CRT group.

\section{Discussion}

With the introduction of cervical screening, the incidence of cervical cancer has dropped dramatically around the world. The disease is expected to decrease further with the expansion of human papillomavirus (HPV) vaccine coverage [19]. Although CT as primary adjuvant therapy for early-stage cervical cancer is not recommended by the National Comprehensive Cancer Network (NCCN) and FIGO, an overwhelming number of women with risk factors for recurrence have accepted postoperative CT in recent years. In this retrospective study, the median follow-up duration for surviving patients was 75 months, the 5-year OS and DFS rates were $86.6 \%$ and $90.0 \%$, respectively. The 5 -year OS were $85.5 \%$ and $87.7 \%$ in the CT and the RT/CRT group, respectively ( $p=0.727)$. There was also no significant difference in the 5-year DSS between the two arms (CT: 87.1\%, RT/CRT: 90.8\%; P=0.548). Our study indicated that CT for early-stage cervical cancer patients meeting Sedlis criteria is a good alternative to RT/CRT as there were no significant differences on rate of recurrence and fewer side effects.

Some studies found that in early-stage cervical cancer patients RS was equivalent to RT in terms of overall and disease-free 5-year survival rate $[20,21]$. In other words, both the treatments were equally effective only in decreasing local recurrences. The main objective of adjuvant therapy after radical surgery, however, ought to be not only to reduce local recurrences but also extra-pelvic recurrences. A number of reports have reported that post-operative RT caused serious complications and relatively higher rate of mortality $[22,23]$. Early leukopenia and gastrointestinal symptoms, late urinary side effects and vaginal shortening or atrophy may occur after RT and CCRT, which can lower the QOL to a considerable extent in cancer patients [24]. Our study also revealed that RT/CRT might add to the spectrum of side effects compared to CT alone. These inferences led us to believe that adopting CT as postsurgical adjuvant therapy might improve the survival rate and QOL in cervical cancer patients. Takeshima et al. reported that a single chemotherapeutic regimen of BOMP (bleomycin, vincristine, mitomycin and cisplatin) improved the 5-year DFS to $93.3 \%$ in patients with intermediate-risk factors and $85.7 \%$ in patients with high-risk factors with adverse events within acceptable range [25]. Lee et al. also revealed statistical difference in the OS between surgery only group and postoperative adjuvant CT group in stage IB cervical cancer with intermediate-risk factors (surgery alone: $81.8 \%$ vs. surgery + CT: $94.9 \%$; $P<0.05$ ) [26]. Lee et al. reported the 3-year DFS and 5-year OS were $94.6 \%$ and $90.6 \%$, respectively rates in 101 patients meeting the Sedlis criteria receiving adjuvant CT alone after RS. This finding indicated that CT should be a rational choice for IB/IIA cervical cancer patients with risk of recurrence [27]. Many studies have compared RT/CRT versus CT to determine better prognosis and fewer complications. A 
previous small sized phase III clinical study reveal that RT+CT was not superior to chemotherapy alone as adjuvant therapy for early-stage cervical cancer patients who are at higher-risk of recurrence after $\mathrm{RH}$ PLND [28]. There are several retrospective studies that concur with this view. Although Hosaka et al. did not indicate the superiority of postoperative CT with BOMP over RT; the authors speculated that using TP might have less adverse effects and confer a survival advantage after radical surgery $[29,30]$. Takekuma et al. certified 4-year PFS and OS did not differ significantly between the CT arm and the CCRT arm in FIGO stage IB1/IIB cervical cancer with high-risk factors who were treated by RS [31]. Similarly, Li et al. showed that 5-year OS and DFS in the CT group were slightly higher than that the group RT in FIGO IB/IIIB cervical cancer [32].

Cervical cancer is one of the most preventable and curable forms of cancer, as long as it is detected early and managed effectively. Given the increase of long-term survivors, the QOL have recently become a crucial issue in early-stage cervical cancer [33,34]. Growing number of researchers began to pay more attention to the adhibition of chemotherapy in early-stage cervical cancer [35, 36, 37, 38]. Previous observations have shown that cervical cancer is moderately chemosensitive. The use of CT for cervical cancer was mainly carried out in recurrent and metastatic cervical cancer. Cisplatin is the most widely used drug; other drugs such as paclitaxel, fluorouracil, bleomycin and carboplatin also are effective in cervical cancer. Over the past decades, paclitaxel in combination with cisplatin has proved to be very effective in advanced cervical cancer [39, 40,41]. An open-label randomized phase III trial JCOG0505 (Japan Clinical Oncology Group) also demonstrated TC was noninferior to TP, with long nonhospitalization periods and similar median OS and PFS [42]. Similarly, Austrian Gynecologic Oncology Group (AGOG) conducted a randomized controlled trial (RCT) in high-risk cervical cancer after $\mathrm{RH}$ to evaluate the impact of chemotherapy [43]. Nevertheless, the application of CT alone as an adjuvant therapy in patients with intermediate-risk cervical cancer has not been fully evaluated.

In 2014, NCCN Guidelines proposed sedlis criteria for external pelvic radiation after RH in node-negative, margin-negative, parametria-negative cases, and is widely accepted as a standard for radiotherapy in early-stage cervical cancer. At the same time, the guidelines pointed out that potentially important risk factors for recurrence may not be limited to the sedlis criteria. Ryu et al. (2014) identified a "four-factor model" (histology, tumour size, deep stromal invasion, and LVSI) in which the presence of any two factors may be useful for predicting recurrence [44]. Other professors also suggested adenocarcinoma (AC) and adenosquamous carcinoma (ASC) are independent prognostic factors (poorer survival outcomes than those with SCC) for cervical cancer patients treated with definitive radiotherapy. In addition, a particular effectiveness of adjunvant RT was seen in reducing the recurrence rate of patients with AC/ ASC vs. squamous cervical tumors [45]. Therefore, it is necessary to distinguish SCC from AC and ASC for discussion $[46,47]$. Our study aim to provide a more targeted treatment strategy for SCC. There are also several limitations of this study. As this was a retrospective study, factors such as heterogeneity of the patients, selection bias and incomplete data may have influenced the results. Besides, absence of patients who met the third category in Sedlis criteria and sub-group analysis of RT and CRT separately may have affected the conclusions to some extent. 


\section{Conclusions}

In summary, our data demonstrated that early-stage cervical cancer patients satisfied to the Sedlis criteria derived clinical benefits from adjuvant CT alone. It suggested that the efficacy of adjuvant chemotherapy alone was at least equivalent, if not superior, to RT/CRT in terms of overall survival, disease-specific survival or recurrence rate with a lower rate of adverse events. As there is no standard systematic chemotherapy regimen for early-stage cervical cancer, further prospective randomized clinical studies on adjuvant chemotherapy in early-stage cervical cancer patients meeting the Sedlis criteria are required to verify the results of this study.

\section{Abbreviations}

RH: radical hysterectomy; RT: radiotherapy; CRT: chemoradiotherapy; CCRT: chemoradiotherapy ; OS: survival rate; RFS: relapse-free survival rate; GOG: Gynecologic Oncology Group; RS: radical surgery; PFS: progression-free survival; QOL: quality of life; CT: chemotherapy; DFS: disease-free survival; TP: Paclitaxel/cisplatin; FIGO: International Federation of Gynecology and Obstetrics; RH-PLND: radical hysterectomy and pelvic lymphadenectomy; SCC: squamous cell carcinoma; LVSI: positive lymphovascular space invasion; TC: paclitaxel/carboplatin; AUC: area under the curve; DSS: diseasespecific survival; WHO: World Health Organization; RTOG: Radiation Therapy Oncology Group; EORTC: European Organization for Research and Treatment of Cancer; HPV: human papillomavirus; NCCN: National Comprehensive Cancer Network; BOMP: bleomycin, vincristine, mitomycin and cisplatin; JCOG: Japan Clinical Oncology Group; AGOG: Austrian Gynecologic Oncology Group; RCT: randomized controlled trial; AC: adenocarcinoma; ASC: adenosquamous carcinoma;

\section{Declarations}

\section{Ethics approval and consent to participate}

All procedures performed in studies involving human participants were in accordance with the ethical standards of the institutional and/or national research committee and with the 1964 Helsinki declaration and its later amendments or comparable ethical standards. This study protocol was approved by the Ethics Committee of Shandong University(KYLL-2018-174). The need for informed consent was waived because of the retrospective nature of the study.

\section{Consent to publication}

Not applicable. 
All data generated or analyzed during this study are included in this published article (and its Supplementary Information files). The datasets are available from the corresponding author upon reasonable request.

\section{Competing interests}

The authors declare that they have no competing interests.

\section{Funding}

This study was funded by: National Natural Science Foundation of China and Science (Grant/Award Number: 81874105); National Clinical Research Center for Gynecological Oncology (Grant/Award Number: 2015BAl13B05); National Natural Science Foundation of China and Science (Grant/Award Number: 81372809) and National Key Research\& Development Program of China (Grant/Award Number: 2016YFC1302903). The funders had no role in study design, in data collection, analysis and interpretation, and in writing the manuscript.

\section{Authors' contributions}

ML: project administration, data analysis, and manuscript writing. YG: project development and data management. YWZ and BW: software, validation. XHY and BXC: data collection, proofread. XSY: manuscript revise, project development. All authors read and approved the final manuscript.

\section{Acknowledgments}

We thank all the people for their work in literature collecting, manuscript compiling , and their help with this work. We would like to express our gratitude to the reviewers and editors.

\section{References}

1. World Health Organization. Human papillomavirus (HPV) and cervical cancer. http://www.who.int/mediacentre/factsheets/fs380/en/. (Accessed January 24, 2019).

2. Koh WJ, Abu-Rustum NR, Bean S, Bradley K, Campos SM, Cho KR, et al. Cervical Cancer, Version 3.2019, NCCN Clinical Practice Guidelines in Oncology. J Natl Compr Canc Netw. 2019;17:64-84.

3. Peters WA, 3rd, Liu PY, Barrett RJ, 2nd, Stock RJ, Monk BJ, Berek JS, et al. Concurrent chemotherapy and pelvic radiation therapy compared with pelvic radiation therapy alone as adjuvant therapy after 
radical surgery in high-risk early-stage cancer of the cervix. J Clin Oncol. 2000;18:1606-13.

4. Sedlis A, Bundy BN, Rotman MZ, Lentz SS, Muderspach LI, Zaino RJ. A randomized trial of pelvic radiation therapy versus no further therapy in selected patients with stage IB carcinoma of the cervix after radical hysterectomy and pelvic lymphadenectomy: A Gynecologic Oncology Group Study. Gynecol Oncol. 1999;73:177-83.

5. Rotman M, Sedlis A, Piedmonte MR, Bundy B, Lentz SS, Muderspach LI, et al. A phase III randomized trial of postoperative pelvic irradiation in Stage IB cervical carcinoma with poor prognostic features: follow-up of a gynecologic oncology group study. Int J Radiat Oncol Biol Phys. 2006;65:169-76.

6. Atkovar G, Uzel O, Ozsahin M, Koca S, Sahinler I, Okkan S, et al. Postoperative radiotherapy in carcinoma of the cervix: treatment results and prognostic factors. Radiother Oncol. 1995;35:198-205.

7. Uno T, Ito H, Itami J, Sato T, Minoura S, Yasuda S, et al. Adjuvant pelvic irradiation in patients with pathologic T2b carcinoma of the uterine cervix. Int J Gynecol Cancer. 2002;12:187-91.

8. $\mathrm{CP} \mathrm{M}, \mathrm{HM} \mathrm{S}, \mathrm{HE} \mathrm{A}$. Is pelvic radiation beneficial in the postoperative management of stage $\mathrm{Ib}$ squamous cell carcinoma of the cervix with pelvic node metastasis treated by radical hysterectomy and pelvic lymphadenectomy? A report from the Presidential Panel at the 1979 Annual Meeting of the Society of Gynecologic Oncologists. Gynecol Oncol. 1980;10:105-10.

9. Hogan WM, Littman P, Griner L, Miller CL, Mikuta JJ. Results of radiation therapy given after radical hysterectomy. Cancer. 1982;49:1278-85.

10. Russell AH, Tong DY, Figge DC, Tamimi HK, Greer BE, Elder SJ. Adjuvant postoperative pelvic radiation for carcinoma of the uterine cervix: pattern of cancer recurrence in patients undergoing elective radiation following radical hysterectomy and pelvic lymphadenectomy. Int J Radiat Oncol Biol Phys. 1984;10:211-4.

11. Kinney WK, Alvarez RD, Reid GC, Schray MF, Soong SJ, Morley GW, et al. Value of adjuvant wholepelvis irradiation after Wertheim hysterectomy for early-stage squamous carcinoma of the cervix with pelvic nodal metastasis: a matched-control study. Gynecol Oncol. 1989;34:258-62.

12. Barter JF, Soong SJ, Shingleton HM, Hatch KD, Orr JW, Jr. Complications of combined radical hysterectomy-postoperative radiation therapy in women with early stage cervical cancer. Gynecol Oncol. 1989;32:292-6.

13. Soisson AP, Soper JT, Clarke-Pearson DL, Berchuck A, Montana G, Creasman WT. Adjuvant radiotherapy following radical hysterectomy for patients with stage IB and IIA cervical cancer. Gynecol Oncol. 1990;37:390-5.

14. Frumovitz M, Sun CC, Schover LR, Munsell MF, Jhingran A, Wharton JT, et al. Quality of life and sexual functioning in cervical cancer survivors. J Clin Oncol. 2005;23:7428-36.

15. Greimel ER, Winter R, Kapp KS, Haas J. Quality of life and sexual functioning after cervical cancer treatment: a long-term follow-up study. Psychooncology. 2009;18:476-82.

16. Iwasaka T, Kamura T, Yokoyama M, Matsuo N, Nakano H, Sugimori H. Adjuvant chemotherapy after radical hysterectomy for cervical carcinoma: a comparison with effects of adjuvant radiotherapy. Obstet Gynecol. 1998;91:977-81. 
17. Monk BJ, Sill MW, McMeekin DS, Cohn DE, Ramondetta LM, Boardman CH, et al. Phase III trial of four cisplatin-containing doublet combinations in stage IVB, recurrent, or persistent cervical carcinoma: a Gynecologic Oncology Group study. J Clin Oncol. 2009;27:4649-55.

18. Cox JD, Stetz J, Pajak TF. Toxicity criteria of the Radiation Therapy Oncology Group (RTOG) and the European Organization for Research and Treatment of Cancer (EORTC). Int J Radiat Oncol Biol Phys. 1995;31:1341-6.

19. Landy R, Pesola F, Castanon A, Sasieni P. Impact of cervical screening on cervical cancer mortality: estimation using stage-specific results from a nested case-control study. $\mathrm{Br} \mathrm{J}$ Cancer. 2016;115:11406.

20. Landoni F, Maneo A, Colombo A, Placa F, Milani R, Perego P, et al. Randomised study of radical surgery versus radiotherapy for stage Ib-lla cervical cancer. Lancet. 1997;350:535-40.

21. Ross Green W, Hathout L, Khan AJ, Elshaikh MA, Beriwal S, Small W, Jr., et al. Revisiting Milan cervical cancer study: Do the original findings hold in the era of chemotherapy? Gynecol Oncol. 2017;144:299-304.

22. Jensen PT, Klee MC, Groenvold M. Validation of a questionnaire for self-rating of urological and gynaecological morbidity after treatment of gynaecological cancer. Radiother Oncol. 2002;65:29-38.

23. Vistad I, Cvancarova M, Kristensen GB, Fossa SD. A study of chronic pelvic pain after radiotherapy in survivors of locally advanced cervical cancer. J Cancer Surviv. 2011;5:208-16.

24. Green JA, Kirwan JM, Tierney JF, Symonds P, Fresco L, Collingwood M, et al. Survival and recurrence after concomitant chemotherapy and radiotherapy for cancer of the uterine cervix: a systematic review and meta-analysis. Lancet. 2001;358:781-6.

25. Takeshima N, Umayahara K, Fujiwara K, Hirai Y, Takizawa K, Hasumi K. Treatment results of adjuvant chemotherapy after radical hysterectomy for intermediate- and high-risk stage IB-IIA cervical cancer. Gynecol Oncol. 2006;103:618-22.

26. Lee TY, Jeung YJ, Lee CJ, Kim HY, Kim SH, Kim WG. Promising treatment results of adjuvant chemotherapy following radical hysterectomy for intermediate risk stage 1B cervical cancer. Obstet Gynecol Sci. 2013;56:15-21.

27. Lee K-B, Kim YS, Lee J-M. Oncologic outcomes of adjuvant chemotherapy alone after radical surgery for stage IB-IIA cervical cancer patients. J Gynecol Oncol. 2018;29.

28. Curtin JP, Hoskins WJ, Venkatraman ES, Almadrones L, Podratz KC. Long H, et al. Adjuvant Chemotherapy versus Chemotherapy plus Pelvic Irradiation for High-Risk Cervical Cancer Patients after Radical Hysterectomy and Pelvic Lymphadenectomy (RH-PLND): A Randomized Phase III Trial. Gynecol Oncol. 1995;61:3-10

29. Hosaka M, Watari H, Kato T, Odagiri T, Konno Y, Endo D, et al. Clinical efficacy of paclitaxel/cisplatin as an adjuvant chemotherapy for patients with cervical cancer who underwent radical hysterectomy and systematic lymphadenectomy. J Surg Oncol. 2012;105:612-6.

30. Hosaka M, Watari H, Takeda M, Moriwaki M, Hara Y, Todo Y, et al. Treatment of cervical cancer with adjuvant chemotherapy versus adjuvant radiotherapy after radical hysterectomy and systematic 
lymphadenectomy. J Obstet Gynaecol Res. 2008;34:552-6.

31. Takekuma M, Kasamatsu Y, Kado N, Kuji S, Tanaka A, Takahashi N, et al. Adjuvant chemotherapy versus concurrent chemoradiotherapy for high-risk cervical cancer after radical hysterectomy and systematic lymphadenectomy. Int J Clin Oncol. 2016;21:741-7.

32. Li S, Hu T, Chen Y, Zhou H, Li X, Cheng X, et al. Adjuvant chemotherapy, a valuable alternative option in selected patients with cervical cancer. PLoS One. 2013;8:e73837.

33. Ashing-Giwa KT, Lim JW, Tang J. Surviving cervical cancer: does health-related quality of life influence survival? Gynecol Oncol. 2010;118:35-42.

34. Fleming ND, Ramirez PT, Soliman PT, Schmeler KM, Chisholm GB, Nick AM, et al. Quality of life after radical trachelectomy for early-stage cervical cancer: A 5-year prospective evaluation. Gynecol Oncol. 2016;143:596-603.

35. Falcetta FS, Medeiros LR, Edelweiss MI, Pohlmann PR, Stein AT, Rosa DD. Adjuvant platinum-based chemotherapy for early stage cervical cancer. Cochrane Database Syst Rev. 2016;11:Cd005342.

36. Kumar L, Gupta S. Integrating Chemotherapy in the Management of Cervical Cancer: A Critical Appraisal. Oncology. 2016;91 Suppl 1:8-17.

37. Matsuo K, Shimada M, Aoki Y, Sakamoto M, Takeshima N, Fujiwara H, et al. Comparison of adjuvant therapy for node-positive clinical stage IB-IIB cervical cancer: Systemic chemotherapy versus pelvic irradiation. Int J Cancer. 2017;141:1042-51.

38. Okazawa-Sakai M, Yokoyama T, Fujimoto E, Okame S, Shiroyama Y, Yokoyama T, et al. Long-term outcomes of postoperative taxane/platinum chemotherapy for early stage cervical cancer: a retrospective study. Int J Clin Oncol. 2018;23:715-25.

39. Kumar L, Pokharel YH, Kumar S, Singh R, Rath GK, Kochupillai V. Single agent versus combination chemotherapy in recurrent cervical cancer. J Obstet Gynaecol Res. 1998;24:401-9.

40. Green JA, Lainakis G. Cytotoxic chemotherapy for advanced or recurrent cervical cancer. Ann Oncol. 2006;17 Suppl 10:x230-2.

41. Cannistra SA, Niloff JM. Cancer of the uterine cervix. N Engl J. Med 1996;334:1030-8.

42. Kitagawa R, Katsumata N, Shibata T, Kamura T, Kasamatsu T, Nakanishi T, et al. Paclitaxel Plus Carboplatin Versus Paclitaxel Plus Cisplatin in Metastatic or Recurrent Cervical Cancer: The OpenLabel Randomized Phase III Trial JCOG0505. J Clin Oncol. 2015;33:2129-35.

43. Lahousen M, Haas J, Pickel H, Hackl A, Kurz C, Ogris H, et al. Chemotherapy versus radiotherapy versus observation for high-risk cervical carcinoma after radical hysterectomy: A randomized, prospective, multicenter trial. Gynecol Oncol. 1999;73:196-201.

44. Ryu SY, Kim MH, Nam BH, Lee TS, Song ES, Park CY, et al. Intermediate-risk grouping of cervical cancer patients treated with radical hysterectomy: a Korean Gynecologic Oncology Group study. $\mathrm{Br} \mathrm{J}$ Cancer. 2014;110:278-85.

45. Rotman M, Sedlis A, Piedmonte MR, Bundy B, Lentz SS, Muderspach LI, et al. A phase III randomized trial of postoperative pelvic irradiation in Stage IB cervical carcinoma with poor prognostic features: 
follow-up of a gynecologic oncology group study. Int J Radiat Oncol Biol Phys. 2006;65:169-76.

46. Noh JM, Park W, Kim YS, Kim JY, Kim HJ, Kim J, et al. Comparison of clinical outcomes of adenocarcinoma and adenosquamous carcinoma in uterine cervical cancer patients receiving surgical resection followed by radiotherapy: a multicenter retrospective study (KROG 13-10). Gynecol Oncol. 2014;132:618-23.

47. Zhou J, Wu SG, Sun JY, Li FY, Lin HX, Chen QH, et al. Comparison of clinical outcomes of squamous cell carcinoma, adenocarcinoma, and adenosquamous carcinoma of the uterine cervix after definitive radiotherapy: a population-based analysis. J Cancer Res Clin Oncol. 2017;143:115-22.

\section{Tables}

Table1

\begin{tabular}{|c|c|c|c|c|}
\hline Characteristic & CT & RT/CRT & Total & P-value \\
\hline Number of patients & 62 & 65 & 127 & \\
\hline Age(mean) & 47 & 47.88 & 47.45 & 0.618 \\
\hline Clinical stage, No.(\%) & & & & 0.382 \\
\hline IB1 & $15(24.2$ & ) $11(16.9)$ & $26(20.5)$ & \\
\hline IB2 & $31(50.0$ & ) $33(50.8$ & $64(50.4)$ & \\
\hline IIA1 & $1(1.6)$ & $5(7.7)$ & $6(4.7)$ & \\
\hline IIA2 & $15(24.2$ & ) $16(24.6)$ & $31(24,4)$ & \\
\hline Sedlis criteria, No.(\%) & & & & 0.811 \\
\hline LVSI+, deep $1 / 3$, any size & $9(14.5)$ & $12(18.5)$ & $21(16.5)$ & \\
\hline LVSI+, middle $1 / 3, \geq 2 \mathrm{~cm}$ & $11(17.7$ & ) $12(18.5)$ & 23(18.1) & \\
\hline LVSI+, superficial $1 / 3, \geq 5 \mathrm{~cm}$ & 0 & 0 & 0 & \\
\hline LVSI-, deep or middle $1 / 3, \geq 2$ & $42(67.7$ & ) $41(63.1)$ & $83(65.4)$ & \\
\hline Grade, No.(\%) & & & & 0.792 \\
\hline Grade 1 & $2(3.2)$ & $2(3.2)$ & $4(3.1)$ & \\
\hline Grade 2 & $22(35.5$ & ) $19(29.2)$ & $41(32.3)$ & \\
\hline Grade 3 & $38(61.3$ & ) $44(67.7)$ & $82(64.6)$ & \\
\hline
\end{tabular}




\section{Table 2}

\begin{tabular}{lccccc}
\hline Site & \multicolumn{2}{c}{ CT (n=62) } & \multicolumn{2}{c}{ RT/CRT (n=65) } & P-value \\
& N & $\%$ & N & $\%$ & \\
\hline no evidence of disease & 54 & 87.1 & 58 & 89.2 & \\
\hline Total recurrence & 8 & 12.9 & 7 & 10.8 & 0.787 \\
\hline local & 5 & 8.1 & 3 & 4.7 & 0.422 \\
\hline Stump & 2 & & 1 & & \\
\hline Pelvis & 3 & & 2 & & \\
\hline Distant & 3 & 4.8 & 4 & 6.2 & 0.745 \\
\hline lung & 1 & & 1 & & \\
\hline Bone & 1 & & 2 & & \\
\hline liver & 1 & 0 & & \\
\hline Paraaortic lymph node & 0 & 1 & & \\
\hline
\end{tabular}

Table3

\begin{tabular}{|c|c|c|c|c|}
\hline Side effects & $\mathrm{CT}(\mathrm{n}=62)$ & $\mathrm{RT} / \mathrm{CRT}(\mathrm{n}=$ & Total $(n=12$ & P-value \\
\hline myelosuppression & $4(6.5 \%)$ & $3(4.7 \%)$ & $7(5.5 \%)$ & 0.650 \\
\hline hepatic toxicity & $9(14,6 \%)$ & $4(6.2 \%)$ & $13(10.2 \%)$ & 0.120 \\
\hline gastrointestinal toxicity & $4(6.5 \%)$ & $1(1.5 \%)$ & $5(4.0 \%)$ & 0.155 \\
\hline urinary disturbance & $0(0.0 \%)$ & $9(13.9 \%)$ & $9(7.1 \%)$ & 0.003 \\
\hline lower-limb lymphedema & $4(6.5 \%)$ & $12(18.5 \%)$ & $16(12.6 \%)$ & 0.041 \\
\hline bowel obstruction & $0(0.0 \%)$ & $3(4.7 \%)$ & $3(2.4 \%)$ & 0.244 \\
\hline
\end{tabular}

\section{Figures}




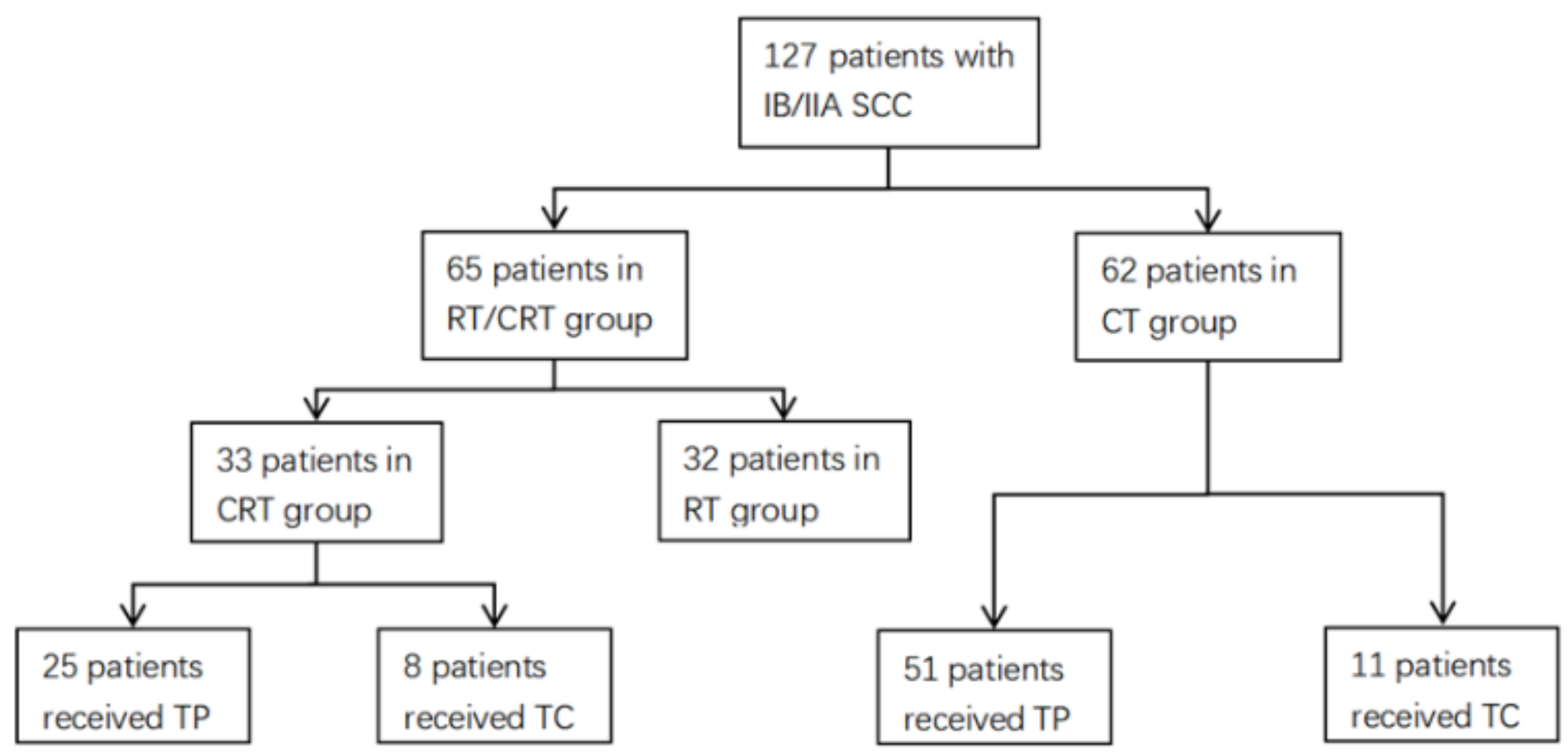

\section{Figure 1}

Chemotherapy details. (SCC: squamous cell carcinoma; RT: radiotherapy; CRT: chemoradiotherapy; CT: chemotherapy; TP: paclitaxel/cisplatin; TC: paclitaxel/carboplatin). 


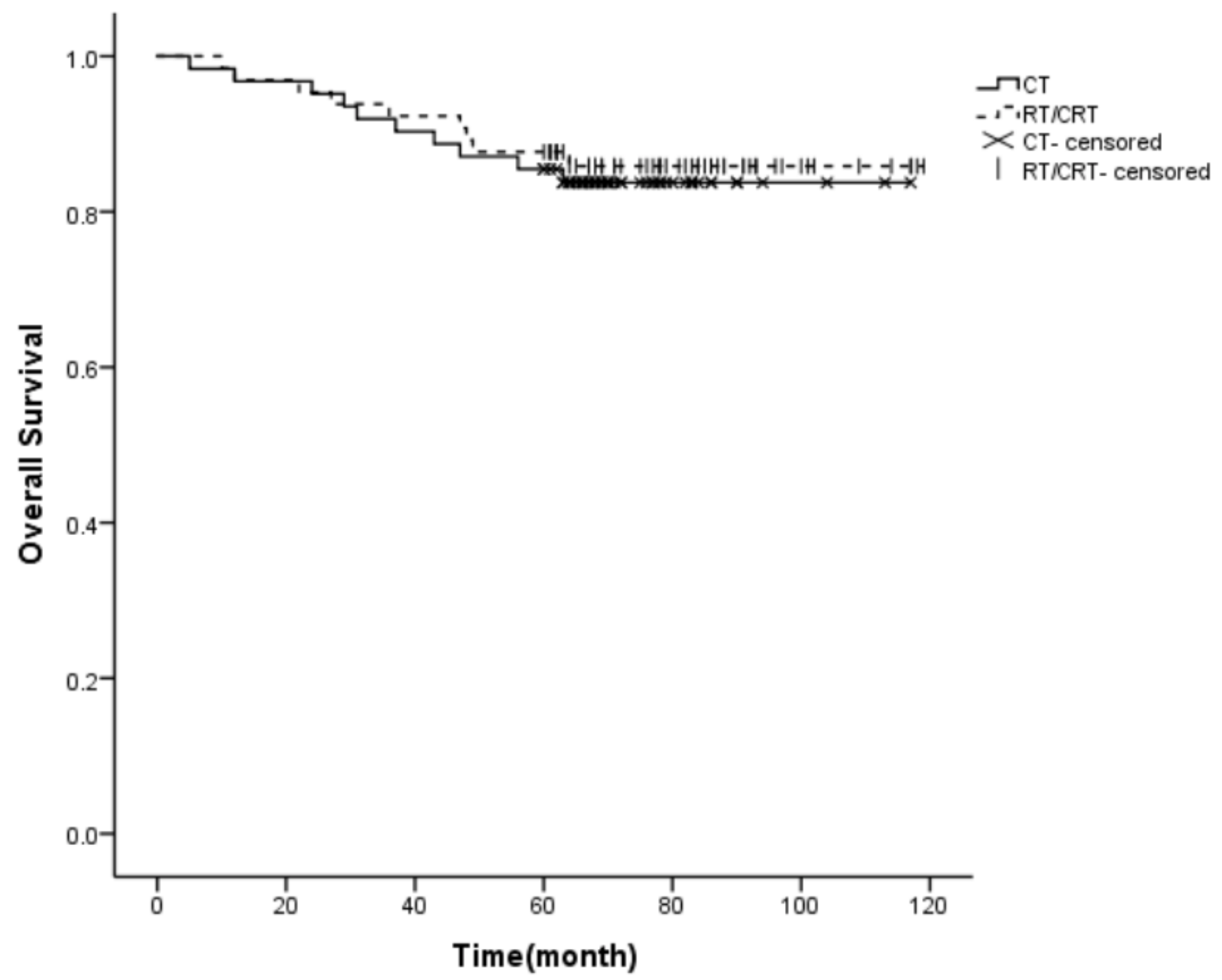

Figure 2

Overall survival of CT $(n=62)$ and RT/CRT $(n=65 ; p=0.727)$. 


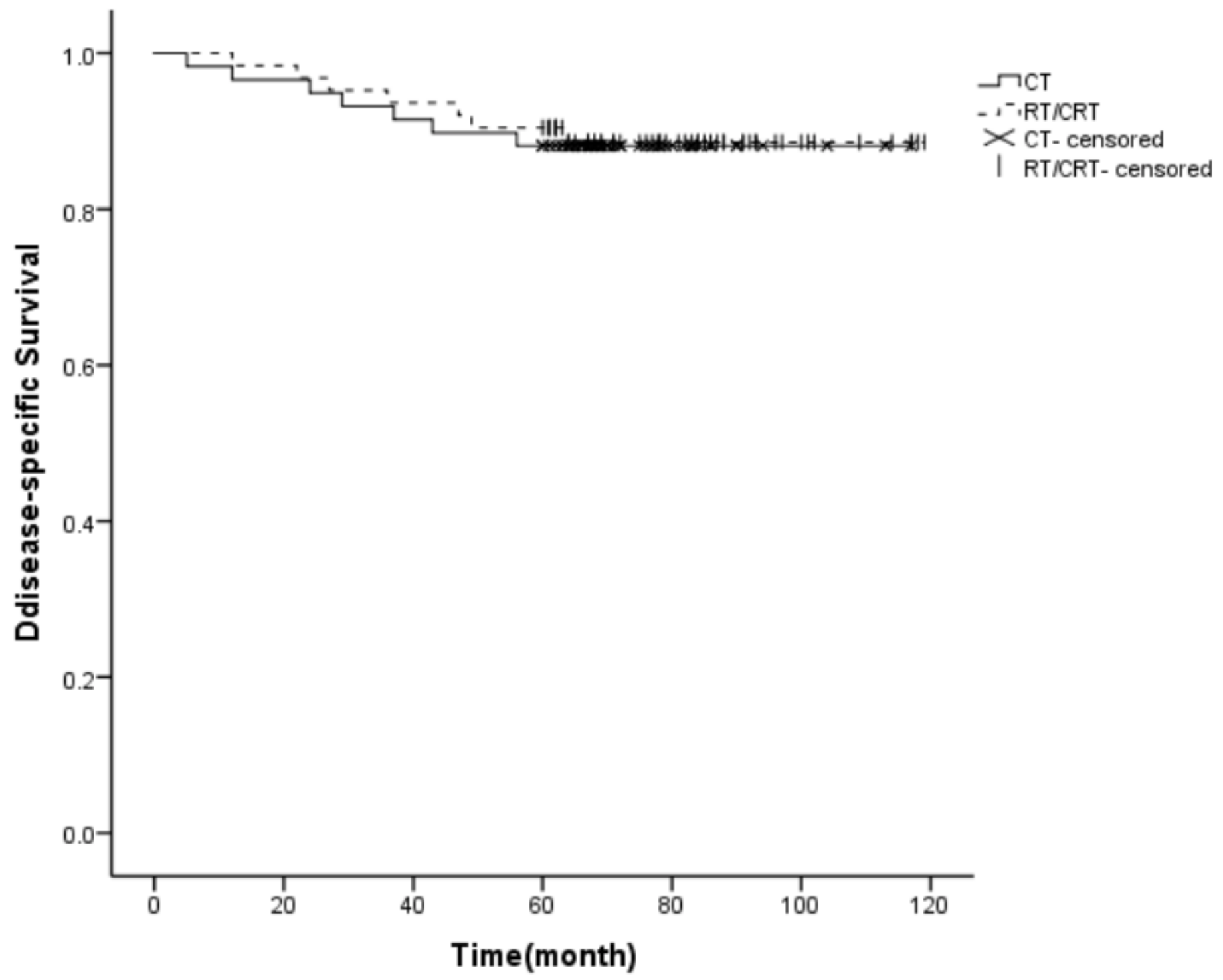

Figure 3

Disease-specific survival of CT $(n=62)$ and RT/CRT group $(n=65 ; p=0.883)$.

\section{Supplementary Files}

This is a list of supplementary files associated with this preprint. Click to download.

- RTorCRT20190709150001.pdf 